\title{
Gamma-Rays as Probes for the Multi-Dimensionality of Type Ia Supernovae
}

\author{
P. Höflich \\ Dept. of Astronomy, University of Texas, Austin, USA
}

\begin{abstract}
We present $\gamma$-ray spectra for a set of Type Ia supernovae models. Our study is based on a detailed Monte Carlo transport scheme for both spherical and full 3-D geometries. Classical and new challenges of the $\gamma$ ray astronomy are addressed. We find that $\gamma$-rays are very suitable to reveal the structure of the envelope and, thus, they allow to probe properties of the nuclear burning front and the progenitor, namely its central density and global asphericities. The potential problems are discussed for the quantitative comparison between theoretical and observed line fluxes during the first few months after the explosion.
\end{abstract}

\section{Introduction}

$\gamma$-ray observations have long been recognized as a potential, valuable tool for supernovae research (Clayton, Colgate \& Fishman, 1969; Ambwani \& Sutherland, 1988; Chan \& Lingenfelter, 1991). Only $\gamma$-rays provide a direct link to the Ni distribution which hardly depends on details of the physics and on the numerical treatment. Different scenarios can be distinguished by line fluxes and profiles, the structure of the progenitors can be probed, and the time of the explosion can be determined. $\gamma$-rays can provide a good determination of the ${ }^{56} \mathrm{Ni}$ production for nearby SNe Ia because, nowadays, accurate distances of nearby galaxies can be obtained by $\delta$ Ceph. Moreover, all sky surveys by $\gamma$-rays may provide an unbiased rate of SNe Ia. As we will discuss below, advances in the fields of optical and IR observations and of the theory have helped to redefine the goals of SN research by $\gamma$-rays. New challenges emerged which emphasize their central role as probes for the 3-D structure of SNe Ia.

The results presented are based on our gamma-ray codes ${ }^{1}$ for spherical (Höflich, Müller \& Khokhlov , 1992) and arbitrary 3-D geometries (Höflich \&

$\square$ available on request

Preprint submitted to Elsevier Preprint 31 October 2018 
1) A typical Delayed Detonation model $(\mathrm{M}(\mathrm{Ni})=0.56 \mathrm{Mo}, \mathrm{M}=\mathrm{Mch})$
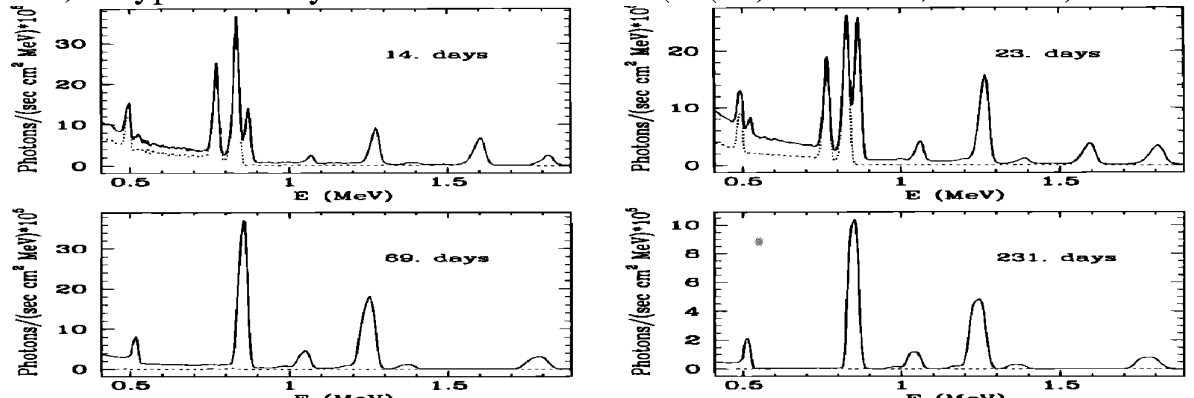

2) $\mathrm{HeD}$ model (M(Ni) $=0.63 \mathrm{Mo}, \mathrm{M}=0.8 \mathrm{M}(\mathrm{C} / \mathrm{O})+0.22 \mathrm{M}(\mathrm{He})$ )
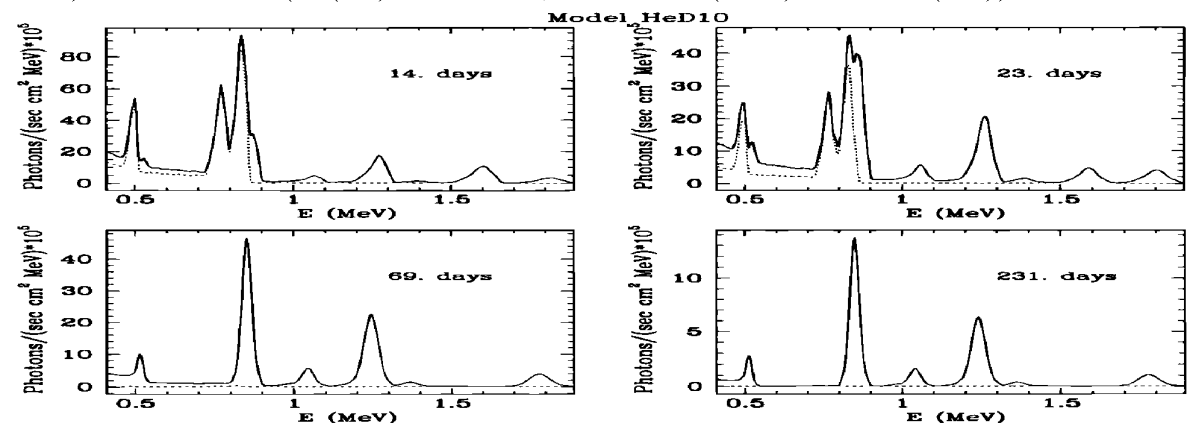

Fig. 1. Comparison of a typical delayed detonation model (DD201) with a helium triggered Sub-Chandrasekhar model (HeD10) (from Höflich, Wheeler J.C. Khokhlov (1998a)). The dashed lines correspond to the contribution of ${ }^{56} \mathrm{Ni}$.

Lee , 2001). We assume homologous expansion of density and chemical structures calculated by spherical and 3-D hydro simulations. All nuclear decay lines of ${ }^{56} \mathrm{Ni}$ and ${ }^{56} \mathrm{Co}$ are included. Pair production and bound-free opacities are taken into account.

\section{Classical and New Challenges}

Classical questions and problems: In parts, this section is based on our previous analyses (Höflich, Müller \& Khokhlov, 1992; Höflich, Wheeler J.C. Khokhlov, 1998a). For further discussions, we also want to refer to Burrows et al. (1991), Kumagai \& Nomoto (1997) and Pinto, Eastman \& Rogers (2001).

Overall, $\gamma$ spectra and their evolution is characterized by a turnover from a phase dominated by ${ }^{56} \mathrm{Ni}$ to ${ }^{56} \mathrm{Co}$ lines (Fig. 1). The time of the explosion can be determined by the ratio between the ${ }^{56} \mathrm{Ni}(0.81 \mathrm{MeV})$ and the ${ }^{56} \mathrm{Co}(0.84$ $\mathrm{MeV}$ ) lines because it varies strongly with time of the explosion but it hardly depends on the model. With time, the envelope becomes increasingly transparent. The spectral evolution depends sensitively on the density and chemical structure of the envelope and, thus, provides a valuable tool for the discrimination of explosion models. For example, sub-Chandrasekhar mass models show as a distinguishing feature an outer layer of ${ }^{56} \mathrm{Ni}$ which reveals itself by high $\gamma$-ray fluxes and broads already a few days after the explosion (Fig. 

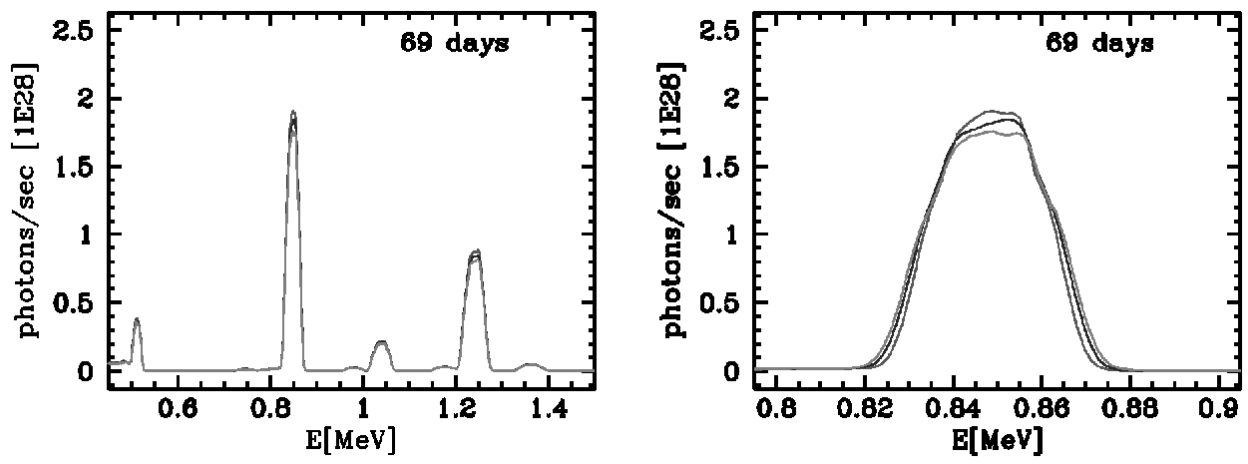

Fig. 2. Influence of the central density on $\gamma$-ray spectra at the example of a delayed-detonation model (Model 5p0z22.20 of Höflich et al. 2001) for $\rho_{c}=2,4 \& 6 \times 10^{9} \mathrm{~g} \mathrm{~cm}^{-3}$ (top to bottom lines).

1). At late phases ( $\geq 100$ days), the absolute line fluxes and profiles provide a direct measure of the total ${ }^{56} \mathrm{Ni}$ mass and its distribution. For $M_{C h}$ models, the high densities close to the center $\left(\geq 10^{9} \mathrm{~g} \mathrm{~cm}^{-3}\right)$ result in the production of neutron-rich iron-group isotopes rather than ${ }^{56} \mathrm{Ni}$. The size of this central region increases with $\rho_{c}$. A knowledge of this property is critical to detect because systematic variations in $\rho_{c}$ can produce an offset (up to $0.2^{m}$, Domínguez, Höflich, \& Straniero (2001)) in the brightness decline relation (Hamuy et al., 1995), a cornerstone of modern cosmology with SNe Ia (e.g. Schmidt et al. (1998); Perlmutter et al. (1999)). Optical and near IR-spectra do not allow to distinguish isotopes. However, the lack of central ${ }^{56} \mathrm{Ni}$ reveals itself in by flat-topped line profiles (Fig. 2). To detect the variation, we need resolutions between 20 to 30 which are well within reach for the upcoming INTEGRAL mission.

We want to mention one problem related to the analysis of observed line fluxes during the first months after the explosion. An advantage of $\gamma$-ray compared to optical analyses is that the results are insensitive to details of the physics or numerical treatment. E.g. the specific opacities do not depend on temperature or density. This advantage is somewhat lost along the way when comparing the observations with theoretical predictions. Problems are caused by the time- and model-dependent line shifts and widths $(\approx 10,000 \mathrm{~km} / \mathrm{s})$, the intrinsic response function of the instrument which is non-Gaussian, and they are connected to the actual definition used to determine integrated line fluxes from synthetic spectra. The actual value may differ by up to a factor of 2 even if based on a given synthetic spectrum (e.g. Müller, Höflich \& Khokhlov (1991)). Preferable, the same machinery should be employed for both the synthetic spectra and the observations to derive accurate values or good upper limits for the $\gamma$ emission.

New challenges: During the last few years, observational and theoretical methods in the field of SN-research advanced significantly well beyond the 

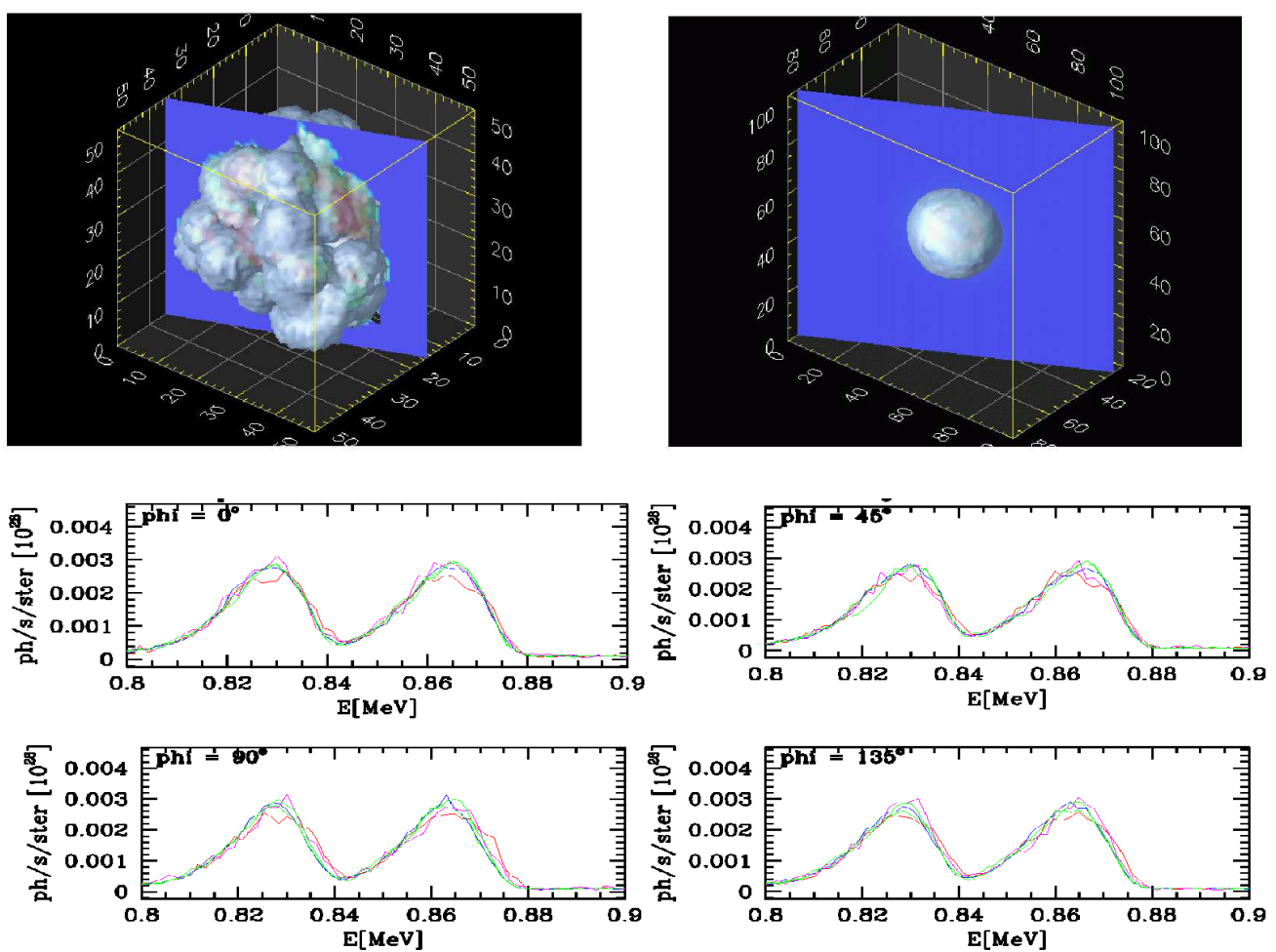

Fig. 3. Energy deposition by $\gamma$-rays at day 1 (left) and 23 (right) based on our full 3-D MC gamma ray transport (Höflich \& Lee, 2001) (upper panels). The diameter of the WD is normalized to 100. In the lower panels, the $\gamma$ spectra at day 23 are given as seen from various $\Theta$ of $90,60,30$ and $0^{\circ}$ (red, blue, green, pink) and $\phi$. In the presence of inhomogeneities, typical fluctuations of about $10 \%$ can be seen. Their frequency provides a measure of the scale. The explosion model is based on the delayed detonation model 5p0z22.20 (2) with $\rho_{c}=2 \times 10^{9} \mathrm{~g} \mathrm{~cm}^{-3}$ assuming chemical inhomogeneities according to Khokhlov (2001).

point imaginable as little as 10 years ago. Back then, SNe Ia were discovered a few days before maximum or later and, in general, light curves were rather uncertain and the spectral coverage was poor. Even for nearby SNe Ia, distances to the host galaxy were uncertain by 20 to $30 \%$. Now, accurate $\delta$-Ceph. based distances of nearby galaxies are available (e.g. Saha et al. (1997)). Combined with the establishment of the optical brightness decline relation relation (Hamuy et al., 1995), SNe Ia provide a unique tool for cosmology. Robotic, systematic SN-search programs (e.g. LOSS, c.f. Treffers et al. (1997)) continuously increase the sample both at low, intermediate and high red-shifts. Often, SNe Ia are discovered 4 to 5 magnitudes before maximum light Riess et al. , 1999; Aldering, Knop \& Nugent, 2000), almost eliminating the selection effects by galactic extinction and providing a tight handle on the rise times. Detailed observations of optical and infrared spectra and light curves allowed sophisticated analyses and test of scenarios, ruling all but out the once popular helium triggered detonations, and strongly favoring deflagration or delayed 
detonation $M_{C h}$ models or, in some instances, the merger scenario (e.g. Höflich \& Khokhlov (1996)). Observation of polarization in SNe Ia has shown that, in general, these objects are fairly spherical (Wang, et al. , 2001) with the noticeable exception of very subluminous SNe Ia (Howell et al., 2001). For the first time, a direct connection with the progenitors seems to be within reach. In particular, there is mounting evidence for a connection between the properties of the progenitor, and the physics of the explosion (Höflich, Wheeler, \& Thielemann, 1998b; Iwamoto et al., 1999; Domínguez, Höflich, \& Straniero, 2001; Khokhlov, 2001). The recent progress may redefine the role of modern $\gamma$-ray astronomy for the field of supernovae, and some of classical goals may have been rendered less compelling. $\gamma$-rays are particular valuable to measure the 3-D structure of SNe Ia. A comprehensive list of new goals is beyond the format of this paper. We want to address two of the areas, namely, $\gamma$-rays as tools to reveal properties of nuclear burning front and large scale asymmetries.

Nuclear burning fronts: Within $M_{C h}$ models, optical and IR LCs and spectra can be reproduced by models in which a (slow) deflagration front turns into a detonation (e.g. Khokhlov (1991)) or, alternatively, a deflagration front is rapidly accelerating as in W7 (Nomoto, Thielemann, \& Yokoi, 1984). However, successful models require parameterized descriptions for the propagation of the burning front. For a discussion, see Domínguez, Höflich, \& Straniero (2001) and references therein. The propagation of a detonation front is well understood but the description of the deflagration and the deflagration to detonation transition (DDT) pose problems. On a microscopic scale, a deflagration propagates due to heat conduction by electrons. Though the laminar flame speed in SNe Ia is well known, the front has been found to be Rayleigh-Taylor (RT) unstable, increasing the effective speed of burning (Nomoto, Sugimoto \& Neo, 1976). Recently, significant progress has been made toward a better understanding of the physics of flames. Starting from static WDs, hydrodynamic calculations of the deflagration fronts have been performed in 2-D (Lisewski et al., 2000), and full 3-D (Khokhlov, 2001). These calculations demonstrated the complicated morphology of the front. Khokhlov (2001) finds that, while the expansion of the envelope becomes almost spherical, the inhomogeneous chemical structure will fill about 50 to $70 \%$ of the star (in mass). The resulting chemical inhomogeneities and their scale depends sensitively on the structure of the initial WD, i.e. progenitor and the pre-conditioning of the runaway. If a DDT occurs at densities needed to reproduce normal-bright $\mathrm{SNe}$ Ia, most of the unburned fuel will be consumed during the detonation phase and, by enlarge, the chemical inhomogeneities will be eliminated. However, they will survive in pure deflagration models.

Currently, state of the art 3-D calculations are restricted to the regime of linear instabilities, i.e. the flamelet regime. The consistent treatment is restricted to the early part of the deflagration phase. In these calculations, The initial acceleration of the the deflagration front is followed by a declining rate of burning. 

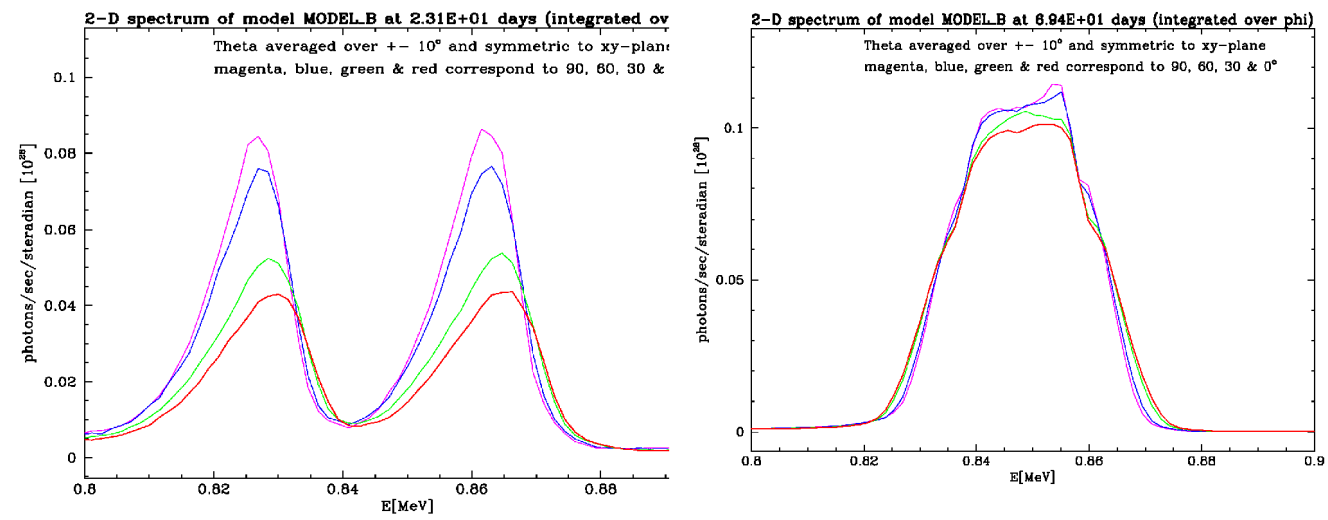

Fig. 4. Directional dependence of the spectrum of an ellipsoidal delayed detonation model (see Fig. 3) at day 23 and 69 after the explosion. We assumed an axis ratio of 0.8 consistent with the optical polarization data for SN1999by.

This leave a significant fraction of the WD unburned $\left(\approx 0.5 M_{\odot}\right)$. The resulting structures cannot account for the observations of typical SNe Ia (Khokhlov. 2001). This problem is well known from spherical deflagration models with a low deflagration speed (e.g. DF1, Khokhlov (1991)), and it triggered the suggestion of a DDT, or, alternatively, showed the need for continuously accelerating deflagration front (Nomoto, Thielemann, \& Yokoi, 1984). Despite the limitations of current models, the chemical clumps will not mix in the subsequent phase. To test for possible effects of chemical inhomogeneities produced during the deflagration, we have remapped a 3-D chemical structure on a spherical explosion model (Fig. 3). The resulting line profiles show fluctuations of about $10 \%$. Their frequency provides a measure of the scale of the instabilities which depends on the $\mathrm{C} / \mathrm{O}$ ratio of the progenitor. Weak or absent fluctuations in the $\gamma$-ray spectra would be a strong indicator for a DDT.

Large scale asymmetries: One of the open questions is the nature of subluminous SNe Ia such as SN 1991bg (Filippenko et al., 1992; Leibundgut et al., 1993). Among them, SN 1999by is one of the best observed SNe Ia. In addition to the studies of optical light curves, detailed polarization spectra of the subluminous SN 1999by have been obtained and analyzed (Howell et al. 2001). Whereas 'normal' SNe Ia tend to show little or no polarization (Wang, Wheeler, \& Höflich, 1997), this supernova was significantly polarized, up to $0.7 \%$, indicating an overall asphericity of the photosphere of $\approx 20 \%$. This result suggests that there may be a connection between the observed asphericity and the subluminosity in SNe Ia. Among others, a possible explanations are the explosion of a rapidly rotating WD and its effect on the propagation of nuclear flames during the explosive phase of burning, or extensive burning of carbon just prior to the runaway (Höflich et al. , 2001). Polarization in the optical measures the asymmetry of the photosphere but, in general, provides little information whether it is caused by a global asymmetry in the density structure or in the excitation mechanism, i.e. the ${ }^{56} \mathrm{Ni}$ distribution. To test the sensitivity of $\gamma$-rays, we have calculated the transport for the subluminous 
model of ellipsoidal shape (Figs. 4). The flux and line profiles vary as a function of inclination (e.g. by $\approx 50 \%$ at day 23 ). In combination with optical and IR observations, $\gamma$-rays can be a key to answer the question on the nature of the asymmetry.

\section{References}

Aldering G., Knop, P., Nugent P. 2000, ApJ , 119, 2110

Ambwani K., Sutherland P.G. 1988, ApJ 325, 820

Burrows A., Shankar A., Van Riper K.A. 1991, ApJ, 379, L7

Chan K.W., Lingenfelter R.E. 1991, 368, 515

Clayton D., Colgate S.A., Fishman G.J. 1969, ApJ 155, 75

Domínguez I., Höflich P., Straniero O. 2001, ApJ ,in press (Aug. 10)

Filippenko A. V. et al. 1992, AJ, 104, 1543

Hamuy, M., Phillips, M. M., Maza, J., Suntzeff, N. B., Schommer, R. A., \& Avilés, R., 1995, AJ, 109, 1

Höflich P., Müller E., Khokhlov A. 1992, A\&A 259, 549

Höflich, P., \& Khokhlov, A. 1996, ApJ 457, 500

Höflich P., Wheeler C.J., Khokhlov A. 1998, ApJ 492, 228

Höflich, P., Wheeler, J. C., \& Thielemann, F. K 1998, ApJ , 495, 617

Höflich P., Gerardy C., Fesen R., Sakai S. 2001, ApJ, submitted

Höflich P., Lee E. 2001, ApJ, in preparation

Howell, A., Höflich, P., Wang, L., \& Wheeler, J. C. 2001, ApJ 556, 302

Iwamoto, K., Brachwitz, F., Nomoto, K., Kishimoto, N., Umeda, H., Hix, W. R., \& Thielemann, F. K. 1999, ApJ s, 125, 439

Khokhlov, A. 2001, ApJ , in press \& astro-ph/0008463

Khokhlov, A. 1991, ApJ , 245, 114

Kumagai K., Nomoto K. 1997, in "Thermonuclear Supernovae", ed. RuizLapuente et al., NATO ASI Ser. C. 486, 515

Leibundgut, B. et al. 1993, AJ, 105, 301

Lisewski, A. M.; Hillebrandt, W.; Woosley, S. E.; Niemeyer, J. C.; Kerstein, A. R. 2000, ApJ 537, 405L

Müller E., Höflich P., Khokhlov A. 1991 248L, 7

Nomoto K., Sugimoto S., \& Neo S. 1976, ApSS, 39, L37

Nomoto, K., Thielemann, F. -K., \& Yokoi, K. 1984, ApJ , 286, 644

Perlmutter S. et al. 1999, ApJ 517, 565

Pinto P., Eastman R.G., Rogers T. 2001, ApJ 551, 231

Riess A. et al. 1999, AJ 118, 2675

Saha A. et al. 1997, ApJ 486, 1

,Schmidt B. P. et al. 1998, ApJ 507, 46

Treffers et al. 1997, IAU-Circ. 7627

Wang, L., Howell, A., Höflich, P., Wheeler J.C. 2001, ApJ 550, 1030

Wang, L., Wheeler, J. C., \& Höflich, P. 1997, ApJ , 476, L27 